with rectal adenocarcarcinoma who recieved long course neoadjuvant chemoradiation followed by surgery were analysed and categorised based on the Tumour Regression Grade(TRG) into 2 groups- Group 1(Good response, TRG 0,1) and Group 2 (Poor response, TRG 2,3). Other factors (clinical and pathological features like lymphovascular/perineural invasion, discontinuous extramural tumour deposits, resection margin status and pTNM stage of tumour) were also evaluated and all variables along with TRG were correlated with disease progression and 5 year survival. Statistical analysis used: IBM SPSS version 20.0 software. Categorical variables expressed using frequency and percentage and the continuous variables presented using mean and standard deviation. The chi-square test was used for finding prognostic factors. Univariate analyses of survival were carried out by Kaplan-Meier method and the evaluations of differences were performed with Log Rank test. Results Group 1 showed reduced risk for disease progression ( $p$ 0.01) and better mean disease free period and overall survival. Poor tumour regression was associated with lymphovascular and perineural invasion and regional lymph node metastases $(\mathrm{p}<0.001)$.

Conclusions Pathological assessment of tumour regression serves as a good predictor for disease outcome and should be assessed in all neoadjuvant treated rectal resection specimens.

\section{PTU-054 MICROSATELLITE INSTABILITY IN STAGE II COLORECTAL CARCINOMA}

Assoc. Prof Roopa Paulose*, Divya Ail, Shital Biradar, K Sundaram. Amrita Institute Of Medical Sciences And Research Centre, Kochi, India

\subsection{6/gutjnl-2018-BSGAbstracts.395}

Introduction $10 \%-15 \%$ of colorectal cancer (CRC) is due to Microsatellite Instability(MSI). The aim of the study was to determine the prognostic significance of detecting MSI in Stage II CRC and to understand the demographic and pathological characteristics of the disease in the Indian scenario.

Methods This was an 8 year retrospective study (2010 to 2017) of 195 patients with Stage II colorectal adenocarcinoma who were categorised into Microsatellite stable (MSS) or MSI based on immunohistochemical expression of the DNA Mismatch Repair(dMMR) proteins. Various clinical and pathological factors as per the Revised Bethseda criteria were compared and analysed between the MSI and MSS groups by Chisquare test and T Test. Kaplan- Meier method was used to calculate the Disease Free Survival (DFS) and Overall-survival (OS) for the 2 groups. Log Rank test was applied to know the strength of association between the DFS and OS with each of the parameters. The data was analysed using IBM SPSS version 20 software.

Results There were 53 (27\%) patients in the MSI group. Younger age, and presence of synchronous or metachronous malignancies, right sided location of tumour, poorly differentiated adenocarcinoma, mucin production and presence of peritumoral Crohn's like lymphocytic response showed statistically significant association with MSI. A definite relationship of MSI status with family history could not be established. The mean DFS in MSI group was $74.7 \pm 3.496$ months as compared to $69.2 \pm 3.631$ months in MSS group. Disease related death was seen in $2.8 \%$ and $15.7 \%$ of patients in MSI and
MSS group respectively, $\mathrm{p}=0.042$. Overall survival among the MSI patients was significantly higher (76.6 44.149 months) than the MSS patients $(65.05 \pm 3.555) \mathrm{p}=0.04$. MSI patients did not show improved survival with adjuvant therapy.

Conclusions Early stage MSI related CRC has good prognosis even without adjuvant chemotherapy. Knowledge of the MSI status in CRC is useful in management decisions and prognostication. In addition it can help to detect those with Lynch Syndrome who may not fulfil the Revised Bethesda criteria.

\section{PTU-055 FLEXIBLE SIGMOIDOSCOPY-BASED ASSESSMENT FOR SUSPECTED CRC - A SERVICE REVIEW OF THE RAPID ACCESS CLINIC}

${ }^{1}$ Aikaterini Peleki*, ${ }^{1}$ Yafit Nahari, ${ }^{1}$ Hasan Jamjoom, ${ }^{1}$ Mahmood Vazirian-Zadeh, ${ }^{1}$ George Flahn, ${ }^{1}$ Raj Patel, ${ }^{1}$ Anthony Kawesha, ${ }^{2}$ Neil Fisher. ${ }^{1}$ Department of General and Colorectal surgery, Russells Hall Hospital, Dudley, UK; ${ }^{2}$ Department of Gastroenterology, Russells Hall Hospital, Dudley, UK

\subsection{6/gutjnl-2018-BSGAbstracts.396}

Introduction The colorectal rapid access clinic (RA) was established in our district general hospital in 2001 to provide a streamlined diagnostic service for patients with suspected colorectal cancer (CRC). It offers direct clinician-led flexible sigmoidoscopy (FS) when appropriate clinical criteria are met (i. e. distal colonic symptoms) as indicated on the GP referral form. Video FS is carried out in JAG-approved facilities in the OP clinic. For many patients this acts as a 'one-stop' clinic and reduces the need for further attendance. The aim of this study was to provide a descriptive review and evaluate patient outcomes over a 4 year period.

Methods A retrospective analysis of electronic records was carried out for all patients undergoing FS as first investigation during the 12 month period of January-December 2013. Demographics, clinical indications, FS diagnosis, further investigations and final diagnosis were recorded and analysed. A subgroup analysis was carried out according to clinical presentation and cancer outcomes were validated with the Somerset CRC database.

Results 1021 patients underwent FS in the RA clinic. Mean age was 72.5 years, 1.1 F:M ratio. The main referral criteria were rectal bleeding (41.8\%), diarrhoea (37.0\%), unspecified altered bowel habit (13.8\%), constipation (7.4\%), abdominal pain $(5.1 \%)$ and weight loss $(2.6 \%)$. Diagnosis at examination was: normal $(24.8 \%)$, diverticulosis $(32.9 \%)$, polyps $(17.5 \%)$, haemorrhoids (26.8\%), malignancy (5.2\%). Further investigations done after FS included barium enema (30\%), colonoscopy (21\%), contrast abdominal CT (35\%) or CT colonogram $(0.5 \%)$. A further 16 cases $(1.5 \%)$ were diagnosed with CRC after full colonic assessment. Discharge rate was $12.9 \%$ after FS and $76.2 \%$ following further investigations. Follow up of patients discharged after RA assessment revealed that four new cases of CRC $(0.4 \%)$ were diagnosed within 3 years of discharge.

Conclusions This unique RA pathway, with FS followed by selective referral for further colonic assessment, offers a quick and reliable service for the exclusion of suspected CRC with excellent diagnostic accuracy. The incidence of interval CRC is within acceptable limits as compared with other colonic imaging. 